\title{
Gradhiva
}

GRADHI

Revue d'anthropologie et d'histoire des arts

$6 \mid 2007$

Voir et reconnaître. L'objet du malentendu

\section{Voir et reconnaître, l'objet du malentendu}

Introduction

\section{(2) OpenEdition}

\section{Journals}

Édition électronique

URL : http://journals.openedition.org/gradhiva/988

DOI : $10.4000 /$ gradhiva.988

ISSN : 1760-849X

\section{Éditeur}

Musée du quai Branly Jacques Chirac

Édition imprimée

Date de publication : 15 novembre 2007

ISBN : 978-2-915133-55-4

ISSN : 0764-8928

Référence électronique

«Voir et reconnaître, l'objet du malentendu », Gradhiva [En ligne], 6 | 2007, mis en ligne le 10 décembre 2008, consulté le 21 septembre 2020. URL : http://journals.openedition.org/gradhiva/988 ; DOI : https://doi.org/10.4000/gradhiva.988

Ce document a été généré automatiquement le 21 septembre 2020

(c) musée du quai Branly 


\title{
Voir et reconnaître, l'objet du malentendu
}

\author{
Introduction
}

1 L'étude des taxinomies, leurs rapports avec l'organisation sociale et plus généralement avec la culture constituent autant de questions classiques en anthropologie. Depuis Mauss, Durkheim ou Lévy-Bruhl, c'est bien de la variabilité culturelle des catégorisations dont il est question. Alors qu'une certaine tradition philosophique recherche depuis Aristote une table universelle des catégories, l'anthropologie vise à rendre compte des différences dans les systèmes de classement, les partitions cognitives structurant la vision occidentale du monde n'étant qu'une modalité parmi d'autres d'appréhension du réel.

2 Face à une "rhapsodie» de systèmes de classification, plusieurs voies ont été explorées. Une première - que l'on pourrait qualifier d'ethnographie catégorielle - vise à restituer les logiques classificatoires au plus près des schèmes autochtones, reposant sur le propre vocabulaire des sociétés étudiées. Elle prend appui sur les critères retenus par une société donnée, telles les qualités associées aux couleurs, aux odeurs, au mode alimentaire, à la parenté, ou encore à la niche écologique, à la hiérarchie politique, etc. C'est à un exercice de traduction dans les termes de sa propre langue que se livre ensuite l'anthropologue. Une autre approche consiste à construire des concepts transculturels fondés sur la comparaison entre taxinomies hétérogènes. Des nomenclatures de parenté ont ainsi été élaborées par la communauté scientifique qui ne vont nullement de soi pour les acteurs concernés, l'alliance et la filiation recouvrant des pratiques variables selon les sociétés.

3 À la fois en affinité et en décalage par rapport à ces deux voies, la question posée dans ce dossier est bien celle de l'adéquation de l'idéel et du réel. Le thème retenu, celui du "voir et reconnaître", privilégie l'étude des malentendus, des méprises, des équivoques. Ces questions sont abordées ici de façon interdisciplinaire. Comment reconnaître un objet, une image, une personne? Quelle est l'origine de ce désajustement des catégories aux choses qu'elles devraient pouvoir circonscrire? Comment se fait malgré tout la reconnaissance, c'est-à-dire l'identificationd'un objet naturel ou social, jusqu'à son inclusion dans une grille conceptuelle préexistante? 
Comment se comporte alors la table des catégories indigènes lorsqu'elle doit intégrer l'inédit, la singularité ou la nouveauté radicale, sans pour autant éclater? Les artefacts, les images, comme les ustensiles importés prennent en effet un sens différent pour les groupes qui entrent en contact, produisant des phénomènes de réinterprétation, notamment en situation coloniale. Si ces équivoques culturelles se produisent fréquemment, l'histoire peut également obscurcir le sens des choses, des objets, des images. Une représentation peut ainsi changer de sens en peu d'années, être recontextualisée ou même altérée à dessein pour remplir une fonction sémantique dans une nouvelle épistémè.

4 C'est à un regard sur le regard que nous convient les auteurs de ce dossier. Ils ont tous en commun de montrer comment les gens, les artefacts et leur représentation sont reconnus ou méconnus. Laurent Barry analyse la construction historique de la naturalité des origines, depuis Ulysse jusqu'au roman moderne. Une épreuve d'authentification est ensuite proposée par Béatrice Fraenkel avec l'invention de l'art pariétal (Europe). Jean-Pierre Goulard, quant à lui, suit le parcours d'une gravure depuis le début du $\mathrm{xIx}^{\mathrm{e}}$ jusqu'au $\mathrm{xx}^{\mathrm{e}}$ siècle, éclairant les transformations qu'elle connaît avec ses publications successives. Puis Dimitri Karadimas décrit les formes de confrontation du monde chrétien et du monde indigène dans le cas d'une figure mythique des Indiens miraña : le Yurupari. La question des catégories de l'aliénabilité et de l'inaliénabilité attachées à des objets sculptés (îles Salomon) est posée par Sandra Revolon dans l'article suivant. Enfin, Gaëlle Beaujan-Baltzer retrace l'itinéraire de plusieurs objets issus du Dahomey depuis leur contexte d'origine jusqu'à leur recontextualisation muséale à l'époque contemporaine. 